\title{
Numerical Analysis of a Flexible Dual Loop Coil and its Experimental Validation for pre-Clinical Magnetic Resonance Imaging of Rodents at $7 \mathrm{~T}$
}

\author{
S. Solis-Najera ${ }^{1}$, F. Vazquez ${ }^{1}$, R. Hernandez ${ }^{1}$, O. Marrufo ${ }^{2}$, and A.O. Rodriguez ${ }^{3}$ \\ ${ }^{1}$ Department of Physics, Faculty of Sciences, National Autonomous University of Mexico, Mexico City, 04510, Mexico, \\ solisnajera@ciencias.unam.mx \\ ${ }^{2}$ Department of Neuroimage, National Institute of Neurology and Neurosurgery MVS, Mexico City14269, Mexico. \\ ${ }^{3}$ Department of Electrical Engineering, Metropolitan Autonomous University Iztapalapa, México City, 09340, Mexico
}

\begin{abstract}
A surface radio frequency coil was developed for small animal image acquisition in a pre-clinical magnetic resonance imaging system at 7 T. A flexible coil composed of two circular loops was developed to closely cover the object to be imaged. Electromagnetic numerical simulations were performed to evaluate its performance before the coil construction. An analytical expression of the mutual inductance for the two circular loops as a function of the separation between them was derived and used to validate the simulations. The RF coil is composed of two circular loops with a $5 \mathrm{~cm}$ external diameter and was tuned to $300 \mathrm{MHz}$ and $50 \mathrm{Ohms}$ matched. The angle between the loops was varied and the Q factor was obtained from the $S_{11}$ simulations for each angle. B1 homogeneity was also evaluated using the electromagnetic simulations. The coil prototype was designed and built considering the numerical simulation results. To show the feasibility of the coil and its performance, saline-solution phantom images were acquired. A correlation of the simulations and imaging experimental results was conducted showing a concordance of 0.88 for the $B_{1}$ field. The best coil performance was obtained at the $90^{\circ}$ aperture angle. A more realistic phantom was also built using a formaldehyde-fixed rat phantom for ex vivo imaging experiments. All images showed a good image quality revealing clearly defined anatomical details of an ex vivo rat.
\end{abstract}

Keywords: Flexible coil, mutual inductance, rat imaging, magnetic resonance imaging.

\section{INTRODUCTION}

Imaging allows systematic assessment of rodent models of a number of human diseases such as cancer, cardiovascular and neurological diseases, and others over the entire disease process, from inception to progression, and monitoring the effectiveness of treatment or other interventions. Each animal serves as its own control and thereby reduces biological variability.

Small animal imaging can be done with magnetic resonance imaging (MRI), magnetic resonance spectroscopic imaging (MRSI), computed tomography (CT), ultrasound (US), and positron emission tomography (PET) [1]. MRI is a noninvasive technique capable of producing images with different contrasts (CNR) of soft tissues and excellent spatial resolution of around $100 \mu \mathrm{m}$, combined with a high temporal resolution. MRI/MRSI may require custom-fabricated coils for specialized applications as well as shimming and other tuning adjustments prior to imaging. Among all the factors affecting the signal-to-noise ratio (SNR) of the MRI experiment [2], the RF coil plays a vital role. The development of RF coils is still a very dynamic and growing area of research in MRI at high and low magnetic fields [3][11].

The development of transceiver coils is mainly focused on the SNR improvement and field uniformity optimization. Transceiver coil performance is a function of the size, shape, excitation signal, and the sample [12]. This motivates the development of transceiver coils with improved SNR and good field uniformity using an adequate and flexible layout to optimally cover the object to be imaged. At higher fields, the magnetic field sensitivity increases approximately linearly but becomes increasingly inhomogeneous. Some effects can be compensated for improved RF coil designs.

The main goal of this paper is to present a flexible surface coil design similar to the butterfly-shaped coil [13] and the paddle coil [14]. Unlike the butterfly coil, our coil design is formed with two circular loops with no crossings. The crossing points cause degradation of the image quality because of the electromagnetic interaction. This layout of the double circular loop coil allows us to cover samples with different geometries and in close proximity. Our coil was operated in the transceiver mode and linearly driven at $300 \mathrm{MHz}$. 
Electromagnetic field (EMF) simulations of our coil were conducted to previous study the properties of the coil magnetic field $\left(B_{1}\right)$. Phantom and ex vivo rat images were acquired to prove its viability to perform imaging experiments of rodents at $7 \mathrm{~T}$.

\section{MEthods \& MATERIALS}

\subsection{COIL DESIGN}

A prototype of the two-circular loop coil was built using a $0.2 \mathrm{~mm}$ thickness copper sheet and each loop had $2 \mathrm{~cm}$ in diameter. The two circular loops joined by corresponding straight segments, as shown in Fig.1.b). From this, it can be observed that no crossings were included in this coil design. Consequently, the magnetic field is not cancelled at the crossing points, and no extra ports are needed to supply the current. This coil feature is the main difference with respect to the butterfly coil design. This particular design aspect allows us to cover more effectively samples with different dimensions. Only samples with similar dimensions to the coil design can be accommodated, see Fig.1.a). This transceiver coil prototype was linearly driven for all imaging experiments. An equivalent circuit was also designed and shown in Fig.1.c)

\subsection{ELECTROMAGNETIC FIELD SIMULATIONS}

The EMF simulation of radio frequency (RF) coils is becoming an increasingly important design tool for applications in high field MRI systems and can be used to optimize the RF coil characteristics for a better performance. Several numerical methods have been developed for the design of RF coils during the last decade improving the study of the RF coil spatial distribution and sensitivity [15]. The electromagnetic characteristics and the spatial distribution of the magnetic field $B_{1}$ produced by our coil were numerically investigated using the finite integral technique (FIT) [16]. The FIT approach has been used for electromagnetic characterization of low and high field MRI RF coils loaded with human-mimicking phantoms with great results [17].

We used the commercial package CST (CST Microwave Studio, CST GmbH, Darmstadt, Germany) because it allows us to run EMF simulations in open boundaries and Perfect Matched Layer (PML) and Perfect Absorbing Boundary (PAB) [18]-[19].

Three-dimensional electromagnetic models of the coil and spherical phantom were designed and used for the numerical simulation (Fig.1.a). A mesh editing for maximum field values was carried out using PAB and particularly extended by the Thin Sheet Technique (TST) to speed up the simulation because mesh lines no longer need to extend through the entire computation domain. The number of lines per wavelength is set to $\lambda / 10$ as a maximum wavelength. For an adjustment between the simulation time and mesh the limit ratio of 10 was used and the space surrounding the entire coil's structure was $125 \mathrm{~mm}$ in Cartesian coordinates. The boundary conditions used were free open space on all the faces of finite volume for minimum reflections.
The dielectric and magnetic properties (Table 1.) for the phantom were considered at $300 \mathrm{MHz}$ [20] and the simulated coil was assumed made of copper. We used a pulse excitation signal of $1 \mathrm{~W}$ in a bandwidth from $270 \mathrm{MHz}$ to $330 \mathrm{MHz}$. The coil was tuned to $300 \mathrm{MHz}$ (proton frequency at $7 \mathrm{~T}$ ) and the coil impedance was $50 \Omega$ for all EMF simulations.

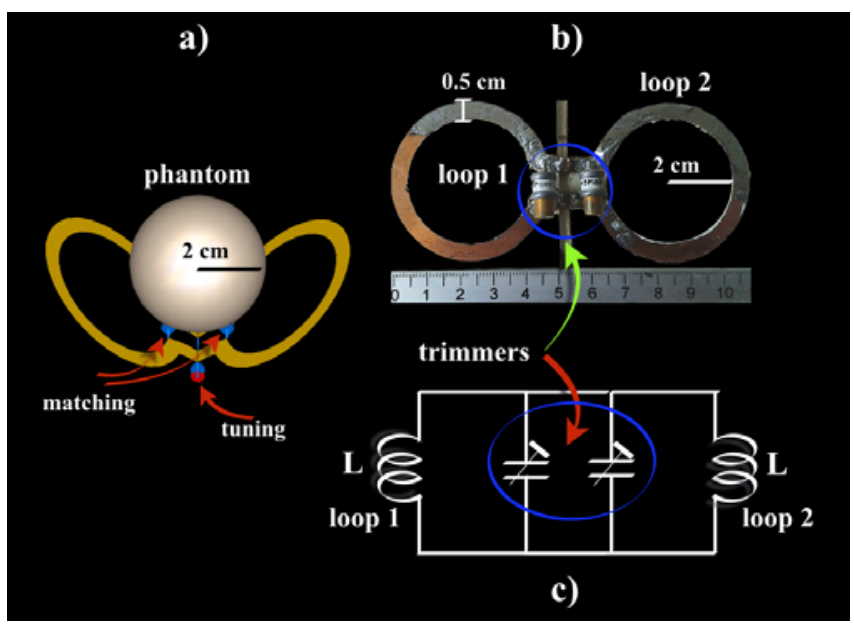

Fig.1. a) Simulation setup with phantom and RF coil. b) Photograph of coil prototype showing dimensions and components. c) Equivalent circuit of coil prototype.

Table 1. Parameters used in the electromagnetic field simulations.

\begin{tabular}{|c|c|c|c|c|}
\hline Element & $\boldsymbol{\sigma}[\mathbf{S} / \mathbf{m}]$ & $\boldsymbol{\varepsilon}$ & $\boldsymbol{\mu}$ & $\tan (\boldsymbol{\delta})$ \\
\hline Air & 0 & 1 & 1 & 0 \\
\hline Copper & $5.8 \times 10^{-7}$ & - & 1 & - \\
\hline Muscle & 0.77052 & 58.201 & 1 & 0.79325 \\
\hline Cortical Bone & 0.21556 & 23.163 & 1 & 0.36855 \\
\hline
\end{tabular}

\subsection{Mutual InduCtanCE THEORY}

Maxwell's equations describe the electric and magnetic fields produced by an RF coil. We used the Biot-Savart law to compute the mutual inductance of the circular loops by finding the magnetic field generated by a constant electric current $I$ in each loop. From Fig.2.a), the magnetic field along $x^{\prime}$ can be expressed as:

$$
B x^{\prime}=\frac{\mu_{0} I a^{2}}{2\left(x^{\prime 2}+a^{2}\right)^{3 / 2}}
$$

where $\mu_{0}$ is the magnetic permeability of vacuum, $a$ is the loop diameter, and $I$ is the electrical current applied to the loops. The distance to any point in the $x$ axis is given by $x=x^{\prime} \cos \beta$. The magnetic field components $B_{x}$ and $B_{y}$ are obtained:

$$
\begin{aligned}
& B_{x}=\frac{\mu_{0} I a^{2}}{2\left[\left(\frac{x}{\cos \beta}\right)^{2}+a^{2}\right]^{3 / 2}} \cos \beta \\
& B_{y}=\frac{\mu_{0} I a^{2}}{2\left[\left(\frac{x}{\cos \beta}\right)^{2}+a^{2}\right]^{3 / 2}} \sin \beta
\end{aligned}
$$



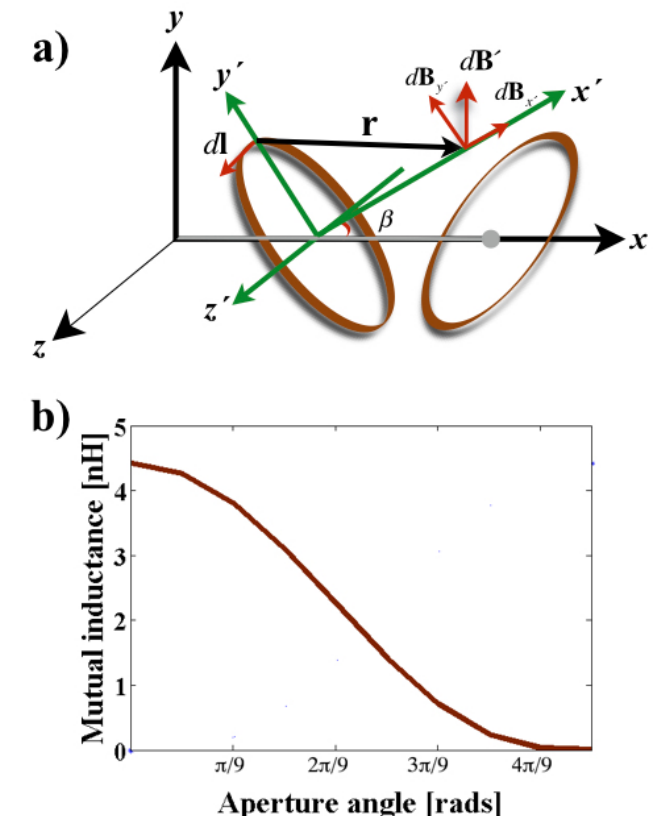

Fig.2. a) Illustration of circular coils inclined at specific aperture angle to compute mutual inductance between the elements. b) Plot of mutual inductance as a function of the aperture angle.

The magnetic field produced by $B_{x}$ and $B_{y}$ components in one loop is $B_{l 1}=\sqrt{B x^{2}+B y^{2}}$. The magnetic flux generated by the first loop induces a magnetic flux in the second loop, $\Phi_{21}=B_{l 1} A_{2}$, with $A_{2}$ being the transversal area over the second loop. Using the reciprocity theorem $\Phi_{12}=B_{l 2} A_{1}$, it is possible to obtain the following expression:

$$
\frac{d \Phi_{21}}{d t}=M_{21} \frac{d I_{1}}{d t}
$$

where $M_{21}$ is the proportional factor that describes the mutual inductance $M$,

$$
M=\frac{\Phi_{21}}{I_{1}}=\frac{\Phi_{21}}{I}
$$

\subsection{EXPERIMENTAL EVALUATIONS}

A $50 \Omega$-coaxial cable was attached to the coil prototype just between the circular loops, and then matched and tuned to $50 \Omega$ and $300 \mathrm{MHz}$, respectively. Only two non-magnetic trimmers (Voltronics, Corp, Cazenovia, NY, USA: 1-10 pF, NMAJ30 0736) were used. Coaxial cables conducted the signal to the low-noise preamplifier. Fig.1.b) shows a coil prototype and its dimensions and electronic components. The resonant frequency was measured using a network analyzer (3753 AH Model, Advantest Co, Tokyo) and return loss $\left(S_{11}\right)$ values were obtained for different aperture angles (Fig.4.).

Images of a spherical phantom and lime were acquired using our coil prototype operated in the transceiver mode and linearly driven. A more realistic phantom was built using a post-mortem Wistar rat (male, 60 g weight, and 30 days old) and it was placed in a cylindrical container filled with formaldehyde solution at $2 \%$ to preserve the tissues and to systematically perform imaging experiments. The cylindrical phantom was made out of acrylic and was $15 \mathrm{~cm}$ long with a $6 \mathrm{~cm}$ diameter. The rat was perfused with a saline solution so the formaldehyde solution can fill all of the cavities between the different tissues and artifacts can be avoided. The Ethics Committee Regulations of the Health and Biological Science Division of Universidad Autonoma Metropolitana-Iztapalapa (Mexico) were followed in all experiments. Fig.8. shows a photo of the phantom. A fast spin echo sequence was used to acquire phantom and lime imaging experiments with the following acquisition parameters: TR/TE $=2000 / 21.71 \mathrm{~ms}$, slice thickness $=1 \mathrm{~mm}$, matrix size $=512 \times 512, \mathrm{FOV}=5 \mathrm{~cm}$ $\mathrm{x} 5 \mathrm{~cm}, \mathrm{NEX}=1$. Ex vivo rat's leg images were also obtained using a standard gradient echo sequence and the following parameters: $\mathrm{TR} / \mathrm{TE}=575 / 8 \mathrm{~ms}$, slice thickness $=1 \mathrm{~mm}$, matrix size $=256 \times 256, \mathrm{FOV}=5 \mathrm{~cm} \times 5 \mathrm{~cm}, \mathrm{NEX}=1$. All the experiments were performed on a $7 \mathrm{~T} / 21 \mathrm{~cm}$ Agilent system equipped with Direct Drive technology (Agilent Technologies, Inc, Santa Clara, CA, USA).

\section{Results AND DisCUSSION}

Simulations of $B_{1}$ were obtained for different aperture angles and are shown in Fig.3. The mutual inductance was calculated from (5) to obtain the worst aperture angle, at $178^{\circ}$ the mutual inductance was $1.42 \times 10^{-57} \mathrm{H}$, while at $90^{\circ}$ it was $2 \mathrm{nH}$. Fig.2.b) shows a theoretical plot of aperture angle-vs.mutual inductance. At $178^{\circ}$ it is possible to obtain images without signal cancelation due to low mutual inductance, but when the angle is decreased, the mutual inductance increases becoming an important factor to be considered.

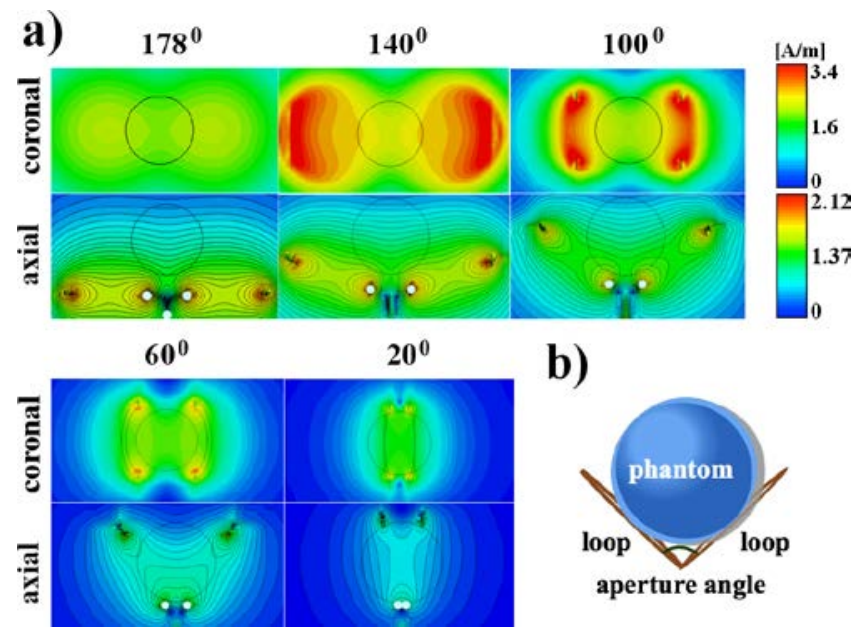

Fig.3. a) Bi-dimensional map series of B1 for different aperture angles. b) Illustration of simulation setup. The black circle in all simulations represents the phantom in b).

Numerical simulations for different coil configurations were used to guide the construction of the final prototypes. The EMF simulations of the bi-dimensional axial and coronal maps of the $B_{1}$ field are shown in Fig.3., for various aperture angles between the circular loops with a spherical phantom. These results confirm that the preliminary coil simulation approach can generate a fairly uniform $B_{1}$ field with an acceptable intensity to obtain MR images. 
To investigate the coil performance for different aperture angles, the simulated $\mathrm{Q}$ factor was obtained from the numerical $S_{11}$ return loss and is shown in Fig.4.

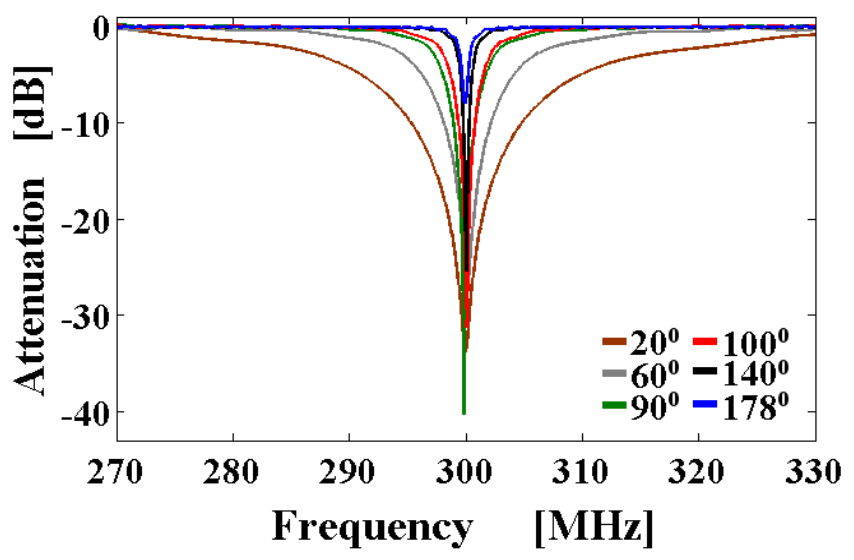

Fig.4. Simulated $S_{11}$-parameter curves of the coil for various aperture angles.

Tuning and matching capacitors were varied to tune the coil at $300 \mathrm{MHz}$. Table 2. summarizes the capacitor values and the $\mathrm{Q}$ factors obtained from the simulated $S_{11}$ parameters. A decrease in the $\mathrm{Q}$ value was observed due to the angle variation. This implies that the coil performance changes in respect to the angle mainly due to the mutual inductance. The values of tuning and matching capacitors were obtained from the numerical simulations in the range $0.1-5.05 \mathrm{pF}$, and were used to calculate the experimental capacitance values.

Table 2. Capacitor values and performance parameters for the coil design.

\begin{tabular}{|c|c|l|l|l|l|}
\hline $\begin{array}{c}\text { Apertur } \\
\text { e Angle } \\
\text { [degrees } \\
\text { ] }\end{array}$ & $\begin{array}{c}\text { Frequenc } \\
\mathbf{y} \\
\text { [MHz] }\end{array}$ & $\begin{array}{c}\mathbf{Q} \\
\text { Factor }\end{array}$ & $\begin{array}{c}\text { Tuning } \\
\text { Trimme } \\
\mathbf{r} \mathbf{[ p F}]\end{array}$ & $\begin{array}{c}\text { Matchin } \\
\mathbf{g} \\
\text { Trimmer } \\
\mathbf{[ p F}]\end{array}$ & $\begin{array}{c}\text { Attenuatio } \\
\mathbf{n} \text { [dB] }\end{array}$ \\
\hline $20^{\circ}$ & 299.94 & 10.77 & 2.28 & 1.45 & -33.69 \\
\hline $60^{\circ}$ & 300.12 & 26.60 & 3.85 & 1.00 & -26.79 \\
\hline $90^{\circ}$ & 299.88 & 58.11 & 4.41 & 0.714 & -40.23 \\
\hline $100^{\circ}$ & 300.06 & 76.93 & 4.44 & 0.623 & -31.29 \\
\hline $140^{\circ}$ & 300.06 & $\begin{array}{l}200.0 \\
4\end{array}$ & 4.87 & 0.384 & -25.36 \\
\hline $178^{\circ}$ & 299.94 & $\begin{array}{l}333.2 \\
6\end{array}$ & 5.05 & 0.100 & -8.025 \\
\hline
\end{tabular}

To fairly study the concordance between the simulations and the phantom images experimentally acquired, a spatial correlation was performed for a specific aperture angle. A concordance coefficient of 0.88 was obtained showing good agreement between the simulation and experimental results.

Changes in frequency as a function of opening angles were investigated, considering 3 cases: $45^{\circ}, 60^{\circ}$, and $178^{\circ}$. The resonant frequency slightly changes with the aperture angle, but tuning and matching can be achieved by adjusting the trimmer capacitors. Fig.5. shows comparison plots of simulated and experimental results.

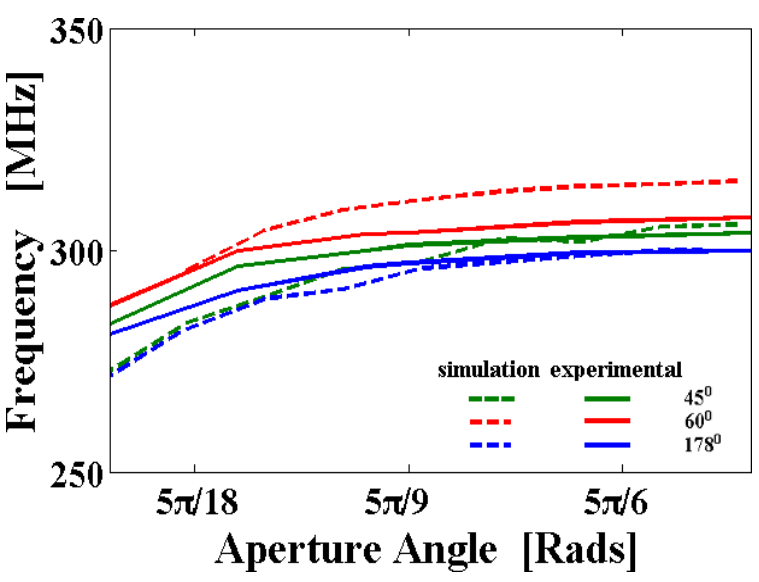

Fig.5. a) Numerical (continuous line) and, b) experiment (dotted line) graphs showing the adjusted frequency due to the aperture angle between the circular loops.

Fig.6. shows images of a spherical phantom and a lime acquired with our coil prototype. For the imaging experiments of the spherical phantom and the lime, the angle between the circular loops was determined by the sample size for each case.

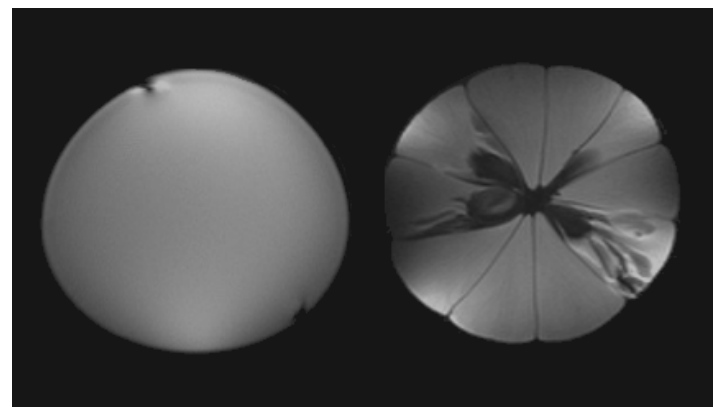

Fig 6. Phantom (left) and lime (right) images with an aperture angle of $40^{\circ}$ and standard pulse sequences. The resolution is 5.1201 pixels/mm and the voxel size: $0.1953 \times 0.1953 \times 1 \mathrm{~mm}^{3}$.

Fig.6. shows image artifacts that may be the cause of proximity of some of the circular loop segments along the coil and around the spherical phantom. This became worse for the lime images where signal loss was evident because both the surface and the radius of the fruit were not even across the samples. The construction imperfections and manipulations of the coil prototype can also cause some image degradation since the circular loops were bent differently for every different sample used. Additionally, the two strips connecting the loops did not produce any signal loss or any other artifact affecting the image quality.

A field uniformity profile was also computed using image data of Fig.6. for comparison purposes and plotted together with the simulation $B_{1}$ field in Fig.3. Uniformity profile comparison shows very good agreement between the experimental and simulation results (Fig.7.). The present discrepancy may be due to proximity of the coil prototype to the spherical phantom and coil manipulation, which are not considered in the simulations. 


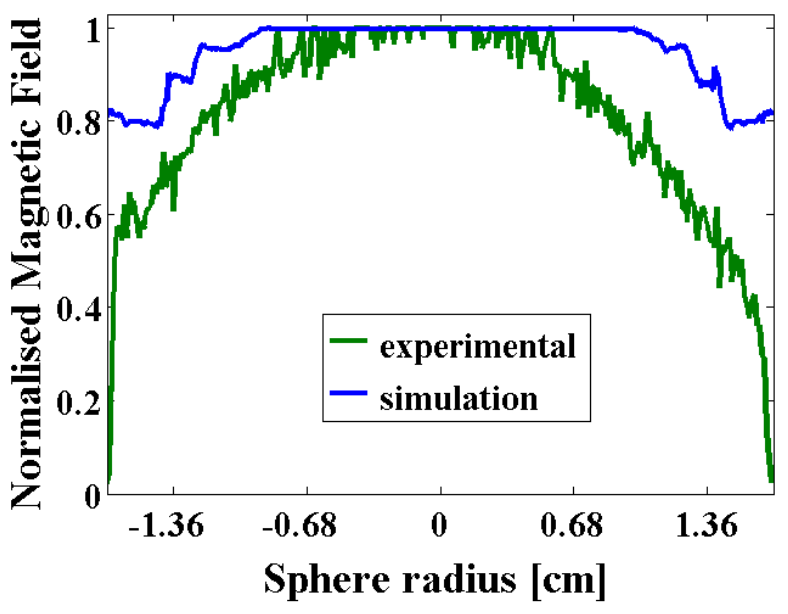

Fig.7. Uniformity profile comparison computed from the EMF simulations and spherical phantom images. Profile was taken along the green line in image phantom.

The SNR of the sphere phantom and lime images are 127 and 60, respectively. The spherical phantom image SNR is twice as much as the lime image SNR, this may be explained by the low content of salts in the sphere.

\section{Ex vivo imaging experiments}

The coil folded around the cylindrical container section, where the ex vivo rat's leg was located to image that particular region of interest, see Fig.8.a). Rat's leg images were then acquired and are shown in Fig.8.c). Good image quality can be observed and the SNR of the rat phantom is 19 . The SNR was computed as mentioned above. A uniformity profile was also computed to investigate the possible effect of the formaldehyde on the image quality, see Fig.8.b).

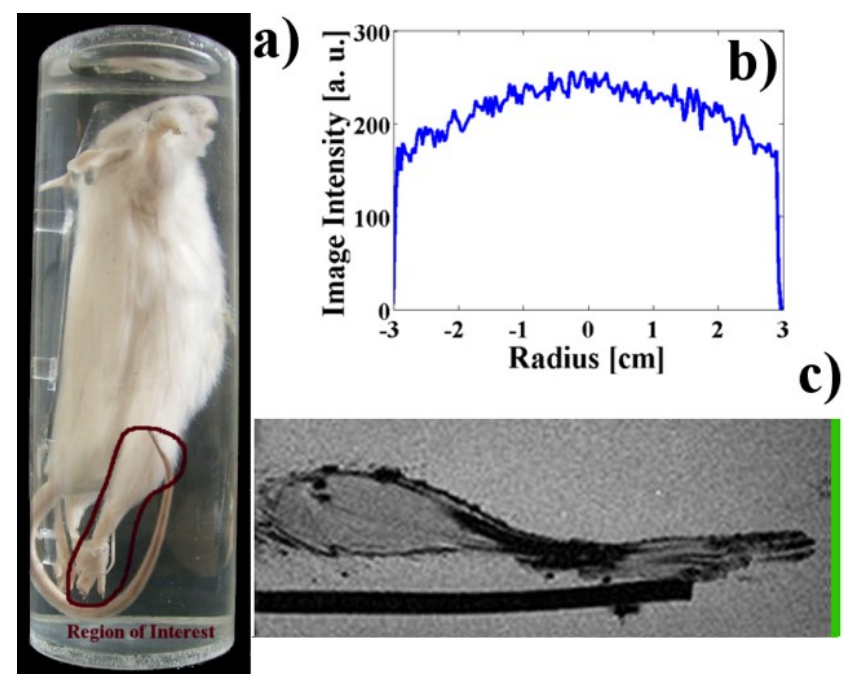

Fig.8. Formaldehyde-fixed rat phantom and ROI a). Uniformity profile b) obtained along the green line in the rat's leg image acquired with the two-circular loop coil c). The resolution for $\mathrm{x}=$ 5.1201 pixels $/ \mathrm{mm}$ and $\mathrm{y}=6.4$ pixels $/ \mathrm{mm}$ and, the voxel size: 0.1953x0.1562x1 $\mathrm{mm}^{3}$.
From these imaging results, there is no clear evidence that the formaldehyde solution affects the image quality and that it creates a good contrast with the rat's leg tissues. The formaldehyde-fixed rat phantom is able to produce more realistic images and it can prove useful for other coil prototypes. This type of phantom can provide a reliable device to test the viability and performance of a number of RF coils for MRI of animal models.

The circular loop dimensions determine the size of the field of view; however, it should be considered that coils with larger dimensions usually suffer from lower SNRs.

\section{CONCLUSIONS}

This paper presents a two-circular loop coil with no crossing elements for high field MRI of rodents. Theoretical and experimental results showed the feasibility of this coil design to generate images of rat's leg and its compatibility with standard pulse sequences. From these results, it can be concluded that the proximity of the two circular loops for samples with similar coil dimensions did not significantly affect the image quality. So it can be safely implied that the magnetic coupling between the circular loops is negligible. Further investigation is necessary to study the SAR levels that may affect the MRI experiments.

\section{ACKNOWLEDGMENT}

S. Solis-Najera would like to thank postdoc stipend from DGAPA-UNAM-Mexico and, PAPIME PE104117 and CONACYT-Mexico Grant No. 112092. We also thank Jorge Kraft and David Flores for assistance with the construction of the formaldehyde-fixed rat phantom and $T$. Bautista for support with numerical simulations.

\section{REFERENCES}

[1] Kiesslin, F., Pichler, B.J. (2011). Small Animal Imaging: Basics and Practical Guide. Springer.

[2] Darrasse, L., Ginefri, J.C. (2003). Perspectives with cryogenic RF probes in biomedical MRI. Biochimie, 85 (9), 915-937.

[3] Childs, A.S., Malik, S.J., O’Regan, D.P., Hajnal, J.V. (2013). Impact of number of channels on RF shimming at 3T. Magnetic Resonance Materials in Physics, Biology and Medicine, 26 (4), 401-410.

[4] Doty, F.D., Entzminger, G., Kulkarni, J., Pamarthy, K., Staab, J.P. (2007). Radio frequency coil technology for small-animal MRI. NMR in Biomedicine, 20 (3), 304325.

[5] Fujita, H. (2007). New horizons in MR technology: RF coil designs and trends. Magnetic Resonance in Medical Sciences, 6 (1) 29-42.

[6] Gilbert, K.M., Curtis, A.T., Gati, J.S., Martyn Klassen, L., Villemaire, L.E., Menon, R.S. (2010). Transmit/receive radiofrequency coil with individually shielded elements. Magnetic Resonance in Medicine, 64 (6), 1640-1651.

[7] Hayes, C.E. (2009). The development of the birdcage resonator: A historical perspective. NMR in Biomedicine, 22 (9), 908-918. 
[8] Novikov, A. (2011). Advanced theory of driven birdcage resonator with losses for biomedical magnetic resonance imaging and spectroscopy. Magnetic Resonance Imaging, 29 (2), 260-271.

[9] Shajan, G., Hoffmann, J., Balla, D., Pohmann, R. (2010). A 700MHz receive array using patch antenna for spin excitation. In Proceedings of the 18th Annual Meeting of ISMRM, May 1-7, 2010, Stockholm, Sweden.

[10] Vaughan, J.T., Griffiths, J.R. (2012). RF Coils for MRI. John Wiley \& Sons.

[11] Xin, X., Han, J., Feng, Y., Feng, Q., Chen, W. (2012). Inverse design of an organ-oriented RF coil for open, vertical-field, MR-guided, focused ultrasound surgery. Magnetic Resonance Imaging, 30 (10), 1519-1526.

[12] Jin, J. (1998). Electromagnetic Analysis and Design in Magnetic Resonance Imaging. CRC Press.

[13] Kumar, A., Bottomley, P.A. (2008). Optimized quadrature surface coil designs. Magnetic Resonance Materials in Physics, Biology and Medicine, 21 (1-2), 41-52.

[14] Constantinides, C., Angeli, S. (2012). Elimination of mutual inductance in NMR phased arrays: The paddle design revisited. Journal of Magnetic Resonance, (222), 59-67.

[15] Stara, R., Fontana, N., Alecci, M., Alfonsetti, M., Galante, A.,Vitacolonna, A., Biagi, L., Buonincontri, G., Del Guerra, A., Tosetti, M., Manara, G., Monorchio, A., Tiberi, G., Retico, A. (2011). RF coil design for low and high field MRI: Numerical methods and measurements. In 2011 IEEE Nuclear Science Symposium and Medical Imaging Conference (NSS/MIC), October 23-29, 2011. IEEE, 3465-3469.
[16] Weiland, T. (1996). Time domain electromagnetic field computation with finite difference methods. International Journal of Numerical Modelling: Electronic Networks, Devices and Fields, 9 (4), 295319.

[17] Solis-Najera, S.E., Martin, R., Vazquez, F., Rodriguez, A.O. (2015). Surface coil with reduced specific absorption rate for rat MRI at 7 T. Magnetic Resonance Materials in Physics, Biology and Medicine, 28, 599608.

[18] Berenger, J.-P. (1996). Three-dimensional perfectly matched layer for the absorption of electromagnetic waves. Journal of Computational Physics, 127 (2), 363-379.

[19] Peterson, A.F., Ray, S.L., Mittra, R. (1997). Computational Methods for Electromagnetics, 1st Ed. Wiley-IEEE Press.

[20] Gabriel, S., Lau, R.W., Gabriel, C. (1996). The dielectric properties of biological tissues: II. Measurements in the frequency range $10 \mathrm{~Hz}$ to $20 \mathrm{GHz}$. Physics in Medicine and Biology, 41 (11), 2251-2269.

Received August 9, 2016 Accepted November 25, 2016 